\title{
Discrimination between healthy and patients with Parkinson's disease from hand resting activity using inertial measurement unit
}

\author{
Luciano Brinck Peres ${ }^{1}$ (i), Bruno Coelho Calil ${ }^{2}$, Ana Paula Sousa Paixão Barroso da Silva ${ }^{3}$, \\ Valdeci Carlos Dionísio ${ }^{4}$, Marcus Fraga Vieira ${ }^{5} \mathbb{B}$, Adriano de Oliveira Andrade ${ }^{1} \mathbb{B}$ and Adriano Alves Pereira ${ }^{*^{*}}$ (D)
}

\begin{abstract}
*Correspondence: adriano.pereira@ufu.br

${ }^{1}$ Postgraduate Program in Electrical and Biomedical Engineering, Faculty of Electrical Engineering, Centre for Innovation and Technology Assessment in Health, Federal University of Uberlândia, Uberlândia, Brazil

Full list of author information is available at the end of the article
\end{abstract}

\begin{abstract}
Background: Parkinson's disease (PD) is a neurological disease that affects the motor system. The associated motor symptoms are muscle rigidity or stiffness, bradykinesia, tremors, and gait disturbances. The correct diagnosis, especially in the initial stages, is fundamental to the life quality of the individual with PD. However, the methods used for diagnosis of PD are still based on subjective criteria. As a result, the objective of this study is the proposal of a method for the discrimination of individuals with PD (in the initial stages of the disease) from healthy groups, based on the inertial sensor recordings.
\end{abstract}

Methods: A total of 27 participants were selected, 15 individuals previously diagnosed with PD and 12 healthy individuals. The data collection was performed using inertial sensors (positioned on the back of the hand and on the back of the forearm). Different numbers of features were used to compare the values of sensitivity, specificity, precision, and accuracy of the classifiers. For group classification, 4 classifiers were used and compared, those being [Random Forest (RF), Support Vector Machine (SVM), K-Nearest Neighbor (KNN), and Naive Bayes (NB)].

Results: When all individuals with PD were analyzed, the best performance for sensitivity and accuracy ( 0.875 and 0.800 , respectively) was found in the SVM classifier, fed with $20 \%$ and $10 \%$ of the features, respectively, while the best performance for specificity and precision ( 0.933 and 0.917 , respectively) was associated with the RF classifier fed with $20 \%$ of all the features. When only individuals with PD and score 1 on the Hoehn and Yahr scale $(\mathrm{HY})$ were analyzed, the best performances for sensitivity, precision and accuracy $(0.933,0.778$ and 0.848 , respectively) were from the SVM classifier, fed with $40 \%$ of all features, and the best result for precision (0.800) was connected to the NB classifier, fed with $20 \%$ of all features.

Conclusion: Through an analysis of all individuals in this study with PD, the best classifier for the detection of PD (sensitivity) was the SVM fed with 20\% of the features and the best classifier for ruling out PD (specificity) was the RF classifier fed with 20\% of the features. When analyzing individuals with PD and score HY=1, the SVM classifier was superior across the sensitivity, precision, and accuracy, and the NB classifier was included in the article's Creative Commons licence and your intended use is not permitted by statutory regulation or exceeds the permitted use, you will need to obtain permission directly from the copyright holder. To view a copy of this licence, visit http://creativecommons.org/ licenses/by/4.0/. The Creative Commons Public Domain Dedication waiver (http://creativecommons.org/publicdomain/zero/1.0/) applies to the data made available in this article, unless otherwise stated in a credit line to the data. 
superior in the specificity. The obtained result indicates that objective methods can be applied to help in the evaluation of PD.

Keywords: Parkinson disease, Inertial sensors, Classifiers, Rest tremor

\section{Background}

Parkinson's disease is the second most common neurodegenerative disease; however, the epidemiological data are not determined precisely [1]. This difficulty can be explained by the different criteria used across the different studies and the precision of the PD diagnosis $[1,2]$. There is a common acceptance that PD possesses a prevalence of approximately $1-2 \%$ of the population over 65 years of age and $0.3 \%$ of the general population [1]. It was estimated that in 2020, PD affected more than 9 million individuals worldwide [3].

The cause of PD is unknown. However, various factors can be considered as being of risk for PD, which include gender (women are marginally more likely to be affected due to longevity), ethnicity (PD possesses greater prevalence in Europe and in North America), genetics, exposure to toxic substances, lethargic encephalitis sequelae, head trauma, and emotional stress [2]. Nevertheless, the main factor of risk is age [4], and as such, it is expected that there will be a drastic increase over the coming years due to the aging of the population [1]. The symptoms of PD can be non-motor or motor. The non-motor symptoms include neuropsychiatric characteristics, dysautonomia, sleep disorder, sensory dysfunction, pain, and fatigue [1]. The motor symptoms that may appear are muscle stiffness, bradykinesia, tremors, and postural imbalance [5], where resting tremor is presented as the main motor symptom (in around $70 \%$ of individuals) [6]. Therefore, PD can have serious impacts on the social and personal life of the patient, which can include incapacity of feeding oneself, drinking water, writing, walking, and even speaking [7].

The impact of PD on life quality, in the decrease in capacity when performing daily routine activities added to prevalence, makes the correct diagnosis of PD essential to outline treatment and measures that can alleviate symptoms, thus improving the ability of individuals to resume their normal daily activities [8]. The correct evaluation of PD can assist clinicians in corrective interventions and improve the life quality of individuals with PD [9], and thus, there are tools for assessing the current stage of PD through use of the Hoehn and Yahr scores [10]. The diagnosing of PD is not a simple task, as the diagnosis can suffer alterations due to the age of the individual and the evolution of symptoms [11]. The most commonly used tool for diagnosing PD is the Unified Parkinson's Disease Rating Scale (UPDRS) [12]. The UPDRS combines a series of clinical scales and questionnaires that have the objective of evaluating the presence and progress of PD motor symptoms [13]. However, the use of UPDRS is more dependent on the professional and, as such, is performed at a certain expense of the patient, as the experience of the professional ends up being a decisive factor when presenting the symptoms at the moment of the diagnosis. In addition, the symptoms of PD can vary due to a number of factors, such as mood, diet, daily habits of patient, absence or not of medication for treating symptoms, and age [14]. One solution to these limitation found in UPDRS could be the use of objective methods [15].

The correct diagnosis of PD is vital for controlling the symptoms and improving the life quality of patients. Although being the most widely used, the UPDRS does have its 
limitations, due to the time consumed in its application and its high degree of subjectivity [12]. An example of the said subjectivity is presented in studies that show a tendency of evaluators to underestimate the severity of the tremor on the least affected side, when the other side presents a severe tremor; this tendency is attributed to less attention being given to the least affected side [16]. The use of an objective method minimizes the need for an experienced professional being involved in the diagnosis process, as it is not always that the patients have such a professional at hand. The challenge takes on greater proportions when assessing individuals with PD in the initial stages of the disease, as is the case of the group of individuals with PD in this study. According to [17], UPDRS is not suitable for assessing PD in its early stages, as most items are related to the more advanced stages of PD.

Due to the aforementioned, there arises the need to discover methods capable of diagnosing PD in a more objective manner. In this sense, the literature presents some proposals using objective methods for the diagnosis of PD [18]. Among these methods, emphasis is placed on those related to the evaluation of movement. This preference is justified by the fact that the symptoms and results of the treatment are manifested through movement in the form of the tremor, bradykinesia, and dyskinesia [7]. The analysis of movement by means of inertial sensors (accelerometers, gyroscopes, and magnetometers) is quite widespread, due to their size and low cost, this allows such instruments to be easily assembled and positioned on different parts of the human body [7].

From the inertial data, one can extract features that will be used for the diagnosis of PD. Currently, there exists a number of studies that propose methods for a more objective diagnosis of $\mathrm{PD}$, using inertial sensors and classifiers. The use of predictors, such as random forest (RF), support vector machine (SVM), K-nearest neighbor (KNN), and Naive Bayes (NB), have helped to differentiate the PD tremor from the essential tremor.

These predictors have been applied to individuals with PD. Research conducted by Kuhner [12] proposes a method to objectively quantify the tremor in individuals with $\mathrm{PD}$, using an RF classifier. The SVM classifier has been used in research to differentiate tremor at rest and postural tremor in individuals with PD from essential tremor in healthy individuals, in addition to being used in research to differentiate the various types of tremor present in individuals with PD [19-21]. The authors in [22] used the KNN for the recognition of daily activities of individuals with PD and healthy individuals, by means of features extracted from inertial sensors. The study conducted in [23] used the NB classifier to diagnose PD, using a PD databank from the UCI machine learning repository.

The above-mentioned classifiers, used in the examples of application concerning individuals with PD, are fed with data that are collected from sensors positioned at strategic points on the individual, through their performing of allocated tasks, but these can sometimes go on to be tiring and as such influence the classification. In this scenario, due to the challenges in diagnosing PD, mainly in the early stages, along with the subjectivity of the main diagnosis method, the introduction of an objective method using inertial sensors for the diagnosis of PD is an area worthy of investigation. Therefore, this study proposes the analysis of feature movements, extracted from inertial sensors, as a tool for diagnosing PD. Our proposal simply uses the hand at rest as a task. In addition, we aim 
to test the performance of different classifiers (RF, SVM, KNN, and NB) to find suitable methods to differentiate the PD group, in the early stages, from the healthy group.

\section{Results}

Additional file 1, Figures S1-S3, illustrate the signals collected from the accelerometer, gyroscope, and magnetometer on the three axes ( $x, y$ and $\mathrm{z}$ ) and the corresponding resultant signal.

The 18 features presented in Table 6 (see section "Methods") were calculated from the signals originating from the sensors, in the same sequence that these are presented in Table 6. As such, a total of 108 features were calculated for the 6 sensors, distributed in the following way: 36 features for the accelerometers, 36 features for the gyroscopes, and 36 features for the magnetometers [the system is composed of two inertial measurement units (IMUs), each IMU possesses an accelerometer, a gyroscope, and a magnetometer].

For all individuals with PD, the highest values for the metric of accuracy were achieved with $10 \%$ of the set of features, and the highest values of the metrics sensitivity, specificity, and precision were achieved with $20 \%$ of the feature set. For individuals with PD and $H Y=1$, the highest values for the metric of specificity was achieved with $20 \%$ of the feature set, and the highest values of the metrics sensitivity, precision, and accuracy were achieved with $40 \%$ of the feature set. As such, Table 1 presents the features for $20 \%$ of the feature set used to feed the classifiers in the analysis of all individuals with PD (the highlight in bold represents $10 \%$ of the features used to feed the classifiers). In addition, Table 1 also presents the features for $40 \%$ of the set of features used to feed the classifiers in the analysis of individuals with PD and $\mathrm{HY}=1$ (the highlight in bold represents $20 \%$ of the characteristics used to feed the classifiers).

Noted on Table 1 is that according to the classification of the most important $20 \%$ of features, only two sensors (accelerometer and gyroscope) are sufficient to feed the classifiers in the analysis of all individuals with PD.

Table 2 shows the results obtained by the classifiers KNN, SVM, RF, and NB in terms of sensitivity, specificity, precision, and accuracy for $10 \%$ of the feature set and $20 \%$ of features for all individuals with PD. The parameters of the classifiers were defined experimentally and those that produced the best response in terms of sensitivity, specificity, precision, and accuracy were chosen. Hence, the classifiers were configured in the following way:

- $\mathrm{KNN}: \mathrm{K}=3$;

- Random Forest: 120 trees;

- Naïve Bayes: kernel (normal);

- Support Vector Machine: polynomial kernel.

From Table 2, one notes that the highest accuracy and sensitivity were achieved with the classifier SVM, fed with $10 \%$ and $20 \%$ of all features, respectively. The highest specificity and precision were achieved with the classifier RF, fed with $20 \%$ of all features.

For individuals with PD and $\mathrm{HY}=1$, the highest values for the metrics sensitivity, precision, and accuracy were achieved with the classifiers fed with $40 \%$ of the feature set and the highest values for the metric of specificity was achieved with the classifiers fed 
Table 1 Features for $20 \%$ and $40 \%$ of the feature set used to feed the classifiers in the analysis of all individuals with PD and individuals with $P D$ and $H Y=1$, respectively

\begin{tabular}{|c|c|c|}
\hline \multirow[t]{2}{*}{ Sensors } & \multicolumn{2}{|l|}{ Features } \\
\hline & $\begin{array}{l}\text { All individuals with PD ( } 20 \% \text { of the feature } \\
\text { set) }\end{array}$ & $\begin{array}{l}\text { Individuals with PD and } \\
\mathrm{HY}=1(40 \% \text { of the feature } \\
\text { set })\end{array}$ \\
\hline \multirow[t]{9}{*}{ Gyroscope 1} & & MAV \\
\hline & Zero crossing & MAVFD \\
\hline & $\mathrm{F} 80^{\mathrm{a}}$ & MAVSD ${ }^{b}$ \\
\hline & EnAp & $\mathrm{RMS}^{\mathrm{b}}$ \\
\hline & EnFuzzy & $\mathrm{PICO}^{\mathrm{b}}$ \\
\hline & SKEW ${ }^{a}$ & Zero crossing \\
\hline & KURTOSIS & $V A R^{b}$ \\
\hline & & RANGE $^{\mathrm{b}}$ \\
\hline & & KURTOSIS \\
\hline \multirow[t]{7}{*}{ Gyroscope 2} & Zero crossing ${ }^{\mathrm{a}}$ & Zero crossing ${ }^{b}$ \\
\hline & $\mathrm{Fpico}^{\mathrm{a}}$ & Fmedia $^{\mathrm{b}}$ \\
\hline & $\mathrm{F} 80^{\mathrm{a}}$ & F50 \\
\hline & EnAp & $\mathrm{F} 80^{\mathrm{b}}$ \\
\hline & EnFuzzy & $E n A p^{b}$ \\
\hline & SKEW & EnFuzzy ${ }^{\mathrm{b}}$ \\
\hline & KURTOSIS & INTQ \\
\hline \multirow[t]{10}{*}{ Accelerometer 1} & & MAV \\
\hline & & MAVFD \\
\hline & & MAVSD \\
\hline & $\mathrm{F} 80$ & $\mathrm{PICO}^{\mathrm{b}}$ \\
\hline & EnAp & Fpico $^{b}$ \\
\hline & SKEW ${ }^{\mathrm{a}}$ & RANGE $^{b}$ \\
\hline & KURTOSIS $^{\mathrm{a}}$ & $\mathrm{INTQ}^{\mathrm{b}}$ \\
\hline & & SKEW ${ }^{b}$ \\
\hline & & KURTOSIS ${ }^{b}$ \\
\hline & & Power3.5-7.5 \\
\hline \multirow[t]{10}{*}{ Accelerometer 2} & & MAV \\
\hline & & MAVFD \\
\hline & & MAVSD ${ }^{b}$ \\
\hline & Zero crossing & RMS \\
\hline & $\mathrm{F} 80$ & Zero crossing ${ }^{b}$ \\
\hline & $E_{A n}{ }^{a}$ & F80 \\
\hline & EnFuzzy $^{\mathrm{a}}$ & EnAp \\
\hline & KURTOSIS ${ }^{a}$ & EnFuzzy \\
\hline & & INTQ \\
\hline & & Power3.5-7.5 \\
\hline \multirow[t]{6}{*}{ Magnetometer 1} & No feature & $\mathrm{F} 50^{\mathrm{b}}$ \\
\hline & & F80 \\
\hline & & EnAp \\
\hline & & EnFuzzy \\
\hline & & VAR \\
\hline & & INTQ \\
\hline \multirow[t]{2}{*}{ Magnetometer 2} & No feature & MAVFD \\
\hline & & INTQ $^{\mathrm{b}}$ \\
\hline
\end{tabular}

a $10 \%$ of the features in the analysis of all individuals with PD

${ }^{b} 20 \%$ of the features in the analysis of individuals with PD and HY $=1$ 
Table 2 Evaluation of the classifiers KNN, SVM, RF, and NB of all individuals with PD

\begin{tabular}{|c|c|c|c|c|c|}
\hline \multirow[t]{2}{*}{ Classifiers } & \multicolumn{4}{|l|}{ Metrics } & \multirow{2}{*}{$\begin{array}{l}\text { Training } \\
\text { accuracy }\end{array}$} \\
\hline & Sensitivity & Specificity & Precision & Accuracy & \\
\hline \multicolumn{6}{|c|}{$10 \%$ of features } \\
\hline KNN & 0.833 & 0.667 & 0.800 & 0.769 & 0.810 \\
\hline SVM & 0.833 & 0.733 & 0.833 & 0.800 & 0.810 \\
\hline RF & 0.667 & 0.800 & 0.842 & 0.718 & 0.690 \\
\hline NB & 0.542 & 0.800 & 0.813 & 0.641 & 0.667 \\
\hline \multicolumn{6}{|c|}{$20 \%$ of features } \\
\hline KNN & 0.750 & 0.667 & 0.783 & 0.718 & 0.738 \\
\hline SVM & 0.875 & 0.600 & 0.778 & 0.769 & 0.738 \\
\hline $\mathrm{RF}$ & 0.458 & 0.933 & 0.917 & 0.641 & 0.690 \\
\hline NB & 0.500 & 0.867 & 0.857 & 0.641 & 0.667 \\
\hline
\end{tabular}

The highest values of the metrics are highlighted in bold

Table 3 Evaluation of the classifiers KNN, SVM, RF, and NB of individuals with PD and with HY=1

\begin{tabular}{llllll}
\hline Classifiers & \multicolumn{3}{l}{ Metrics } & & $\begin{array}{l}\text { Training } \\
\text { accuracy }\end{array}$ \\
\cline { 2 - 4 } & sensitivity & Specificity & Precision & Accuracy & \\
\hline 20\% of features & & & & & \\
KNN & 0.667 & 0.556 & 0.556 & 0.606 & 0.722 \\
SVM & 0.733 & 0.611 & 0.611 & 0.667 & 0.639 \\
RF & 0.600 & 0.444 & 0.474 & 0.515 & 0.722 \\
NB & 0.533 & $\mathbf{0 . 8 3 3}$ & 0.727 & 0.697 & 0.583 \\
40\% of features & & & & & \\
KNN & 0.733 & 0.556 & 0.579 & 0.636 & 0.778 \\
SVM & $\mathbf{0 . 9 3 3}$ & 0.778 & $\mathbf{0 . 7 7 8}$ & $\mathbf{0 . 8 4 8}$ & 0.750 \\
RF & 0.533 & 0.778 & 0.667 & 0.667 & 0.611 \\
NB & 0.667 & 0.722 & 0.667 & 0.697 & 0.583 \\
\hline
\end{tabular}

The highest values of the metrics are highlighted in bold

with $20 \%$ of the feature set. Table 3 shows the results obtained by the classifiers KNN, SVM, RF, and NB in terms of sensitivity, specificity, precision, and accuracy for $40 \%$ and $20 \%$ of the feature set.

The comparison of the values found on Tables 2 and 3 shows that when only individuals with PD with $\mathrm{HY}=1$ are considered, the performance of the classifiers is better in relation to the metrics sensitivity and accuracy, but the metrics specificity and precision are lower in relation to the group that uses all individuals with PD.

\section{Discussion}

\section{Evaluation of extracted features}

The signal that produces the inertial sensor recordings, arising from Parkinson's disease, possesses various proposals for its evaluation. The signal from inertial sensor recordings in PD can be evaluated through features related to frequency $[24,25]$, and other features related to amplitude, signal entropy, form of data distribution, and variability can also provide important features when analyzing the signal from inertial sensor recordings in 
individuals with PD [24]. Amplitude is linked to a scalar measurement (negative or positive) in the oscillation of a movement [26]. Additionally, some studies show the use of entropy can aid in the classification process of other diseases [27].

There were 18 features chosen that allow for the classification of individuals as healthy or with PD. We used 5 features related to amplitude (RMS, PEAK, MAV, MAVFD, and MAVSD), 6 feature related to frequency (Zero Crossing, Fmedia, Fpico, F50, F80, and Power3.5_7.5), 2 features related to signal entropy (ApEn and FuzzyEn), 3 features related to signal variability (VAR, Range, and IntlA), and 2 features related to data distribution (Skewness and Kurtosis).

By analyzing all individuals with PD, the groups of features Amplitude and Variability were not considered important for the calculation of the metrics sensitivity, specificity, precision, and accuracy. The features of frequency, form of data distribution and entropy stood out in this differentiation in relation to sensitivity and accuracy. This observation was already expected to some extent, as participants with PD were in early stages and without any clear signs of tremor.

\section{Evaluation of classifiers}

This study compared the KNN, SVM, RF, and NB classifiers, KNN is currently used as a benchmark for this type of application, and the SVM, RF and NB classifiers are widely used today, which means that this comparison can aid the PD diagnosis in becoming more effective [28-30].

The study performed in [31] also used the inertial sensors of accelerometer and gyroscope, but applied to gait of those with PD alongside healthy individuals, to differentiate the relative parameters for gait between the two groups. The classifiers used were also, among others, the KNN, SVM, RF, and NB, and the parameter used for validating the result was accuracy, the classifier that presented the best general result was the KNN with an accuracy of $84.5 \%$. The collaborators in [32] analyzed the gait of individuals with PD alongside healthy individuals, by means of a force platform. The classifiers used in the present study were also used, among others, in the study by [32]. The best accuracy obtained by the authors was with the SVM classifier of around $90 \%$, followed by the methods RF and KNN with an accuracy around $87 \%$.

Our study agrees with that in [32], as in our study, the best performance in relation to accuracy, in terms of the comparison between classifiers was presented by SVM in both analysis, when analyzing all individuals with $\mathrm{PD}$, along with the analysis of individuals with $\mathrm{PD}$ and $\mathrm{HY}=1$ ( $80 \%$ and $85 \%$ respectively).

The four statistical metrics used (sensitivity, specificity, precision, and accuracy) possess the capacity of demonstrating the performance of the classifiers. Our study showed a better performance for SVM classifier in the sensitivity and accuracy metrics. When analyzing all individuals with PD, the best performance was found in the RF classifier in relation to the specificity and precision metrics. When analyzing individuals with PD and $\mathrm{HY}=1$, the best performance was found in the NB classifier in relation to specificity and the best performance in relation to the precision metric was found in the SVM classifier.

The accuracy found in our study was similar that presented in the results of [31, 32]. Despite the metrics calculated in our work being close to the values found in [31,32], it 
is important to highlight the statement of [33], where emphasis is placed in their work on the performance comparison of the classifiers among several studies, which must be carried out carefully, due to the differences involved in the calculation of the metrics, such as the parameters of the algorithms and the features used.

\section{Percentage of features}

Despite not being the objective of the article, the behavior of the classifiers in relation to the percentage of the features used was analyzed. Figure 1 shows the performance of the classifiers when all individuals with PD are analyzed. Figure 1 shows that when all individuals with PD were analyzed together, the best performance in relation to the metrics sensitivity and accuracy is presented by the SVM classifier, when using $20 \%$ and $10 \%$ of the feature set, respectively. Regarding the metrics specificity and precision, the best performance was presented by the RF classifier using $20 \%$ of the feature set, only features derived from the accelerometer and gyroscope sensors were needed for classification.

Figure 2 shows the performance of the classifiers with individuals suffering from PD with the score $\mathrm{HY}=1$ being analyzed. In this analysis, the best performance is presented by the SVM classifier in regards to the metrics sensitivity, precision, and accuracy, using $40 \%$ of the feature set. The best performance in relation to specificity was the NB classifier using $20 \%$ of the feature set, and all sensors were needed for classification (accelerometers, gyroscopes, and magnetometers).

\section{Evaluation of the results for the PD groups}

Two groups of individuals with PD were evaluated in comparison to the healthy group, and the first group evaluated was composed of all individuals with PD (HY scores 1 and 2). The other group analyzed were individuals with PD with $\mathrm{HY}=1$. The results for the metrics sensitivity and accuracy were superior in the analysis of the group of individuals with $H Y=1$. This result can be explained by the presence of

(a) Sensitivity

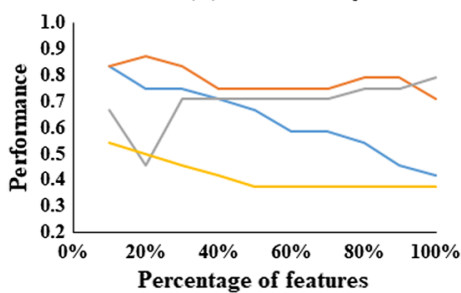

(c) Precision

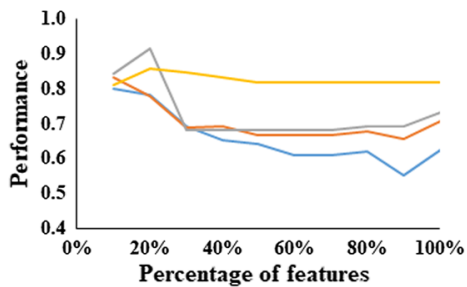

(b) Specificity

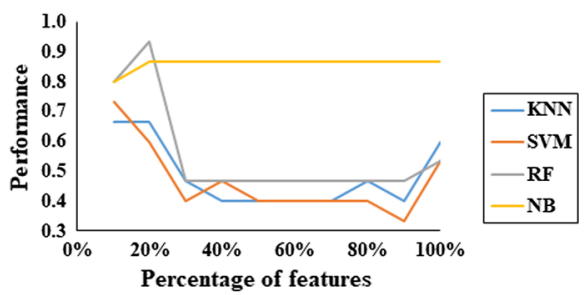

(d) Accuracy

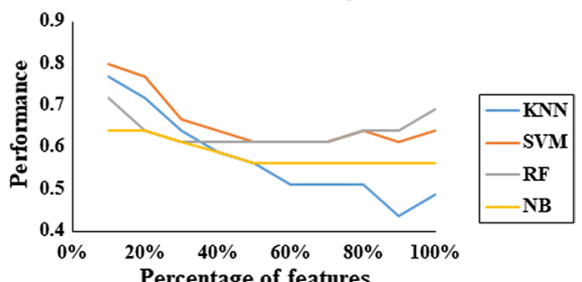

Fig. 1 Classifier performance in relation to the number of features of individuals with PD with score $H Y=1$ and 2. Metrics: a sensitivity, $\mathbf{b}$ specificity, $\mathbf{c}$ precision, and $\mathbf{d}$ accuracy 
(a) Sensitivity

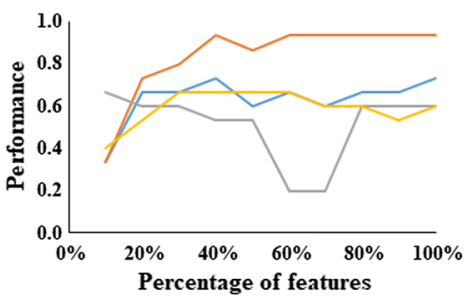

(c) Precision

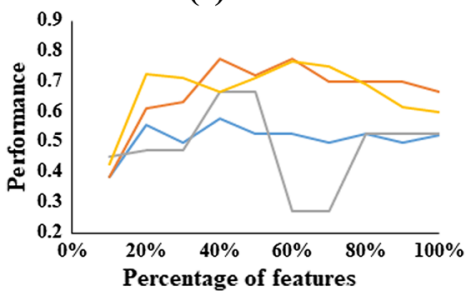

(b) Specificity

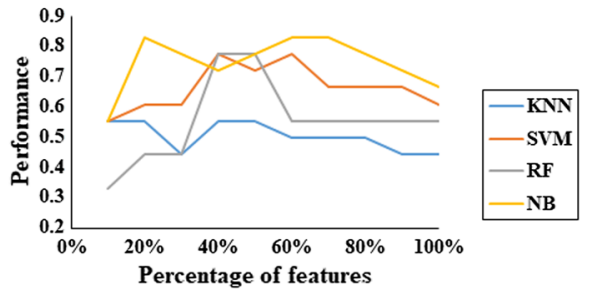

(d) Accuracy

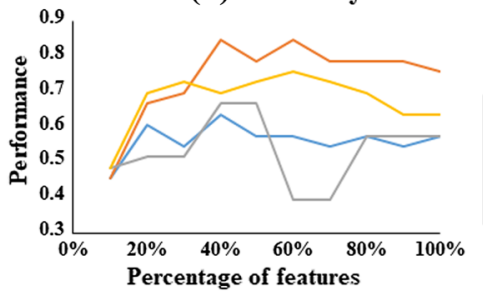

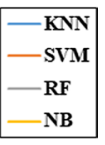

NB

Fig. 2 Classifier performance in relation to the number of features of individuals with PD and HY $=1$. Metrics: a sensitivity, $\mathbf{b}$ specificity, $\mathbf{c}$ precision, and $\mathbf{d}$ accuracy

individuals with more evident tremor in the first analysis $(\mathrm{HY}=2)$, these individuals possess features that are very much separate from the other participants in the PD group, leading to an approximation of the features between the healthy group and the individuals with PD that present lesser oscillations measured from the inertial sensors.

Noteworthy here is that the individuals in our sample showed signs of Parkinson`s in the early stages, usually stages that are difficult to diagnose. Success in differentiation suggests that these classifiers are promising for differentiating between individuals with PD from healthy individuals. Therefore, we encourage future studies that investigate the success rate of classifiers to differentiate the groups (PD and healthy) with large samples, as well as their ability to classify bradykinesia.

\section{Limitations}

The main limitations of this study are the number of participants and the absence of a guideline to define the features used and the positioning of the IMUs. Furthermore, our work classified the groups of subjects with Parkinson's disease and the group of healthy subjects based on inertial sensors. The group of subjects with Parkinson's disease was in the early stages of the disease, so that 11 of the 15 subjects with Parkinson's disease did not show visible signs of tremor. However, the signals collected by the inertial sensors had oscillations that allowed for the classification of the analyzed groups. Thus, although $70 \%$ of the subjects with PD experience tremors [6], there are a number of people with PD who do not experience tremor or it is not the first symptom presented, in these cases, the technique presented is not able to classify the groups. In addition, the only symptom analyzed was the oscillations present in the signal, and other symptoms should be considered to improve the accuracy of the method for the proper classification of the groups studied. 


\section{Conclusion}

When we analyzed all patients with PD, our study suggests that for the calculation of accuracy in the evaluation of PD, only 11 features, comprising of accelerometers and gyroscopes, possess the best performance. When the objective of the evaluation is sensitivity, specificity, and precision, the best performance was achieved using $20 \%$ of all the features calculated in this study. The results for the metrics sensitivity and accuracy in the analysis of individuals with PD and $\mathrm{HY}=1$ were superior to the results found when we used all individuals with PD in this study. The obtained result indicates that objective methods can be applied in the evaluation of PD. The statistical data from this study are in agreement with the literature. The use of computational and objective methods aids in evaluating the signal of the inertial sensors of the individual performing the hand at rest task, while mitigating the effect of the variations of the symptoms. The technique proposed in this study allows for the automatic classification of individuals and can be used to aid in diagnosing patients with suspected PD, even in the initial stages of the disease.

\section{Methods}

\section{Ethical aspects}

This study was approved by the Human Research Ethics Committee (HERC-n² 270.782) of the Universidade Federal de Uberlândia, and by the National Ethics Research Committee (NERC $-\mathrm{n}^{\circ} 361.526$ ) of the National Health Council.

\section{Participants}

The subjects with Parkinson's disease were recruited from the ambulatory facility of the Hospital de Clínicas of Uberlândia of the Federal University of Uberlândia (HCU-UFU), at the physiotherapy clinic of the Federal University of the Triângulo Mineiro (Uberaba), and at the Parkinson association of the Triângulo Mineiro in Uberlândia.

Twenty-seven individuals from both genders were enrolled in this study, across the age group of 50 years or over. From these, 12 individuals were healthy and 15 diagnosed with PD (Table 4), in the initial stage of PD (stages 1 and 2 of the Hoehn and Yahr score (HY) [10]). The participants were divided into 2 groups, with the first group being made up of individuals with $\mathrm{PD}\left(S_{\mathrm{PD}}\right)$, and the second group of the healthy individuals $\left(\mathrm{S}_{\mathrm{H}}=12\right.$ individuals).

In this study, two analyses were performed. Initially, the classification between groups was carried out with healthy individuals and all individuals with $\mathrm{PD}\left(S_{\mathrm{PD}}=15\right.$ individuals). The second analysis consisted of the classification between healthy individuals and the group composed of individuals with $\mathrm{PD}$ with $\mathrm{HY}=1\left(S_{\mathrm{PD}}=11\right.$ individuals). The data

Table 4 Demographic characteristics of the participants of this study

\begin{tabular}{lll}
\hline Characteristics & $S_{\text {PD }}$ & $S_{\mathbf{H}}$ \\
\hline Number of subjects & 15 & 12 \\
Age (years) (mean \pm SD) & $65.3 \pm 9.1$ & $60.1 \pm 6.1$ \\
Gender/number of subjects & $\mathrm{M} / 8-\mathrm{F} / 7$ & $\mathrm{M} / 4-\mathrm{F} / 8$ \\
Hand Analyzed /number of subjects & $\mathrm{R} / 9-\mathrm{L} / 6$ & $\mathrm{R} / 12$ \\
\hline
\end{tabular}

$M$ male, $F$ female, $R$ right, $L$ left, $S D$ standard deviation 
collection was performed during the period "ON" of the individuals with PD. The subjects with PD were diagnosed by a neurologist.

Table 5 shows the tremor score according to the UPDRS scale part 3 (motor section) item 20 (tremor at rest) and the HY score of all PD volunteers.

\section{Data collection}

Data collection was performed using the device TREMSEN (Precise Tremor Sensing Technology, INPI: BR 102014023282 6) developed by researchers from the Center for Innovation and Technological Evaluation in Health [Núcleo de Inovação e Avaliação Tecnológica em Saúde (NIATS)] from the Federal University of Uberlândia. TREMSEN is composed of a gyroscope (L3GD20H, STMicroelectronics, Switzerland), an accelerometer and a magnetometer. The sensibility of the gyroscope, accelerometer, and of the magnetometer was configured to $\pm 245^{\circ} / \mathrm{s}, \pm 2 \mathrm{~g}$ and \pm 2 gauss respectively, in accordance with the studies of [29].

The signals collected were digitalized at $50 \mathrm{~Hz}$, using a microcontroller (Atmel SAM3X8E ARM Cortex-M3) with a 12-bit resolution digital/analog converter. The data were stored on a laptop by means of serial communication, the software used was developed for TREMSEN in C\# (Microsoft). The data were stored in text format and processed by a custom code written in R-Studio.

Parkinson's disease generates a complex set of movements, which is notorious for producing uncontrollable jerking movements of the forearm, wrist, and hand. Due to this fact, two sets of Inertial Measurement Unit (IMUs) were used. The IMU 1 was positioned on the back of the hand, the IMU 2 was positioned on the back of forearm, between 3 and $4 \mathrm{~cm}$ from the wrist joint, with the direction of the axes oriented according to Fig. 3. On the healthy individuals and individuals with PD without showing obvious signs of tremor (all individuals with $\mathrm{PD}$ with $\mathrm{HY}=1$ ), the inertial sensor was

Table 5 Score of UPDRS (part 3 item 20) and HY score of all PD volunteers across the study population

\begin{tabular}{lll}
\hline Volunteer & $\begin{array}{l}\text { UPDRS } \\
\text { (part 3, item 20) }\end{array}$ & Score HY \\
\hline 1 & 1 & 1 \\
2 & 0 & 1 \\
3 & 3 & 2 \\
4 & 1 & 1 \\
5 & 0 & 1 \\
6 & 2 & 2 \\
7 & 2 & 2 \\
8 & 0 & 1 \\
9 & 1 & 1 \\
10 & 1 & 1 \\
11 & 0 & 1 \\
12 & 1 & 1 \\
13 & 1 & 1 \\
14 & 2 & 2 \\
15 & 0 & 1 \\
\hline
\end{tabular}




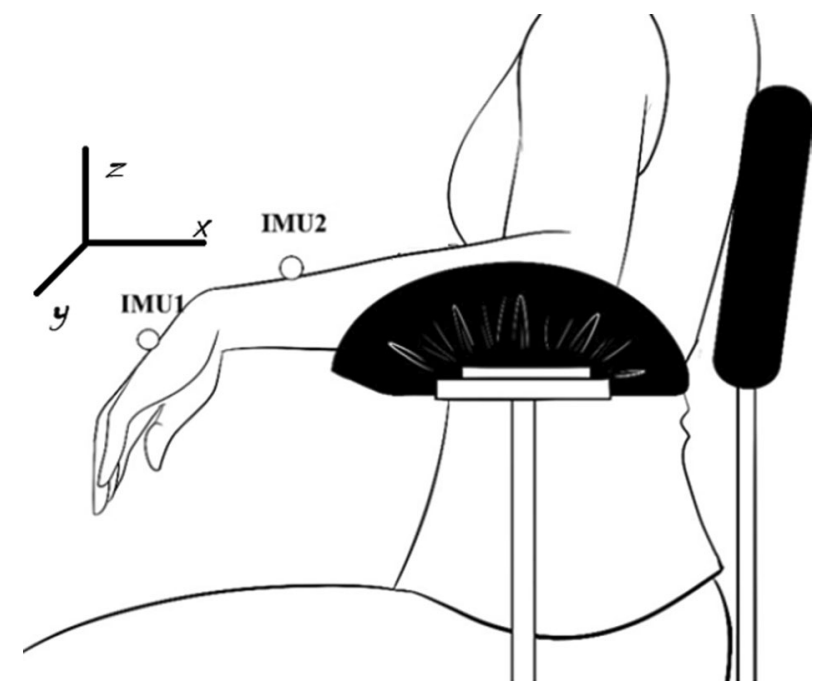

Fig. 3 Positioning of the subject and inertial sensor unit, as well as the orientation of inertial sensor axes

positioned on the dominant hand (Fig. 3), while on the individuals with PD and tremor, the sensor was positioned on the hand most affected by the tremor (four individuals with PD with $\mathrm{HY}=2$ ).

The data were collected from the participants in accordance with [29]. The subjects maintained the wrist at rest and pendant for a short period, the forearm supported on a rest with the hand pendant, forearm in pronation, and the palm of the hand facing downwards. The measurements were collected three times, with intervals of $60 \mathrm{~s}$, where each collection was comprised of $15 \mathrm{~s}$.

\section{Signal preprocessing}

The signals were band-pass-filtered between 1 and $16 \mathrm{~Hz}$ to remove low- and high-frequency artifacts [34].

The start and end of tasks were marked manually by pressing a push-button, generating a pulse of $15 \mathrm{~s}$ (manual pulse). Following this, the resultant of the 3 axes of the accelerometer, gyroscope, and magnetometer sensors were calculated, using Eq. (1):

$$
R=\sqrt{x^{2}+y^{2}+z^{2}}
$$

where $x, y$, and $z$ are the measurements from the sensors along their respective axes and $R$ is the resultant.

For the removal of linear trends, the value of $R$ was subtracted from its mean. The resulting signal was used to calculate the features.

\section{Extraction of features}

The features used in this study are described in Table 6.

108 features were extracted from each participant, considering 18 features for each sensor (accelerometers, gyroscopes and magnetometers from IMUs 1 and 2). 
Table 6 Features extracted from the signals

\begin{tabular}{|c|c|c|}
\hline Feature & $\begin{array}{l}\text { Source of the } \\
\text { features }\end{array}$ & Definition \\
\hline Root mean square (RMS) & [35-38] & $\begin{array}{l}\text { RMS }=\sqrt{\frac{1}{N} \sum_{n=1}^{N} x(n)^{2}} \\
\text { where } N \text { is the number of elements of } X \\
\quad\left(X=\left\{x_{1}, x_{2}, \ldots, x_{n}\right\}\right) ; x(n) \text { is the } n \text {th element }\end{array}$ \\
\hline Peak & [35] & Maximum value of the signal \\
\hline Mean absolute value (MAV) & {$[35,36,39]$} & $\begin{array}{l}\text { The patterns are organized into windows and the } \\
\text { average value of each window is used as the } \\
\text { value of the feature } \\
M A V=\frac{1}{5} \sum_{m=1}^{S}\left|X_{m}\right| \\
S \text { is the number of samples per window; } X_{m} \text { is the } \\
\text { m-th sample of the window }\end{array}$ \\
\hline $\begin{array}{l}\text { Mean absolute value of the first differ- } \\
\text { ence (MAVFD) }\end{array}$ & {$[35,40,41]$} & MAVFD $=\frac{1}{N-1} \sum_{n=1}^{N-1}|x(n+1)-x(n)|$ \\
\hline $\begin{array}{l}\text { Mean absolute value of the second dif- } \\
\text { ference (MAVSD) }\end{array}$ & {$[35,40]$} & MAVSD $=\frac{1}{N-2} \sum_{n=1}^{N-2}|x(n+2)-x(n)|$ \\
\hline Mean frequency (FMEAN) & $\begin{array}{l}{[35,36,38,42,} \\
43]\end{array}$ & $\begin{array}{l}\text { FMEAN }=\frac{\sum_{n=1}^{N}\left(P_{n}(n) * f_{n}(n)\right)}{\sum_{n=1}^{N} P_{n}(n)} \\
\text { where } P_{n} \text { is the Power spectrum; } f_{n} \text { is the vector } \\
\text { frequency of } P_{n ;} N \text { is the number of samples }\end{array}$ \\
\hline Zero crossing (ZC) & {$[35-37,39]$} & Computes how many times the signal crosses zero \\
\hline Peak frequency (FPEAK) & {$[42-44]$} & $\begin{array}{l}\text { FPEAK is a frequency at which the maximum power } \\
\text { occurs } \\
\text { FPEAK = maximum }\left(P_{n}\right)\end{array}$ \\
\hline Median frequency (F50) & $\begin{array}{l}{[35,36,38} \\
42-44]\end{array}$ & $\sum_{n=1}^{F 50} P_{n}(n)=\sum_{F 50}^{N} P_{n}(n)=\frac{1}{2} * \sum_{n=1}^{N} P_{n}(n)$ \\
\hline $\begin{array}{l}\text { Frequency for which } 80 \% \text { of the total } \\
\text { power of } P_{n} \text { is below this value (F80) }\end{array}$ & {$[43,45]$} & $\sum_{n=1}^{F 80} P_{n}(n)=0.8 * \sum_{n=1}^{N} P_{n}(n)$ \\
\hline $\begin{array}{l}\text { Power in frequency band } 3.5-7.5 \mathrm{~Hz} \\
\text { (Power3.5_7.5) }\end{array}$ & [46] & Power3.5_7.5 $=\sum_{f_{n}=3.5}^{f_{n=7.5}} P_{n}(n)$ \\
\hline Approximate entropy (ApEn) & $\begin{array}{l}{[35,37,45,47,} \\
\quad 48]\end{array}$ & $\begin{array}{l}\text { According to [48]: } \\
\text { - For a time series of sample } N\{u(1), u(2), u(3) \ldots u(N)\} \\
\text { given } m \text {, forms sequences of vectors } x(1) \text { through } \\
x(N-M+1), \text { defined by } x(i)=\{u(i), u(i+1), \ldots, u \\
(i+m-1)\}, i=1, \ldots, N-m+1 ; \\
\text { - Compute the distance between the vectors } x(i) \\
\text { and } x(j) \text { defined as the maximum difference } \\
\text { between each element of the vectors }(d[x(i), x(j)]) \text {; } \\
\text { - For each } i \leq N-m+1, \text { com- } \\
\text { pute } C_{i}^{m}(r) \text {, which is defined as: } \\
\text { (numberofjsuchasd }[x(i), x(j)] \leq r) /(N-m+1) ; \\
\text { - Define: } C^{m}(r)=(N-m+1)\left(\sum_{i=1}^{N-m+1} \ln C_{i}^{m}(r) \text {; }\right. \\
\text { - The approximated entropy is defined by: } \\
\text { ApEn }(m, r, N)=C^{m}(r)-C^{m+1}(r) \\
\text { where } m \text { is the length of the comparative window; } \\
r \text { is the tolerance; In is the natural logarithm }\end{array}$ \\
\hline
\end{tabular}


Table 6 (continued)

\begin{tabular}{|c|c|c|}
\hline Feature & $\begin{array}{l}\text { Source of the } \\
\text { features }\end{array}$ & Definition \\
\hline Fuzzy entropy (FuzzyEn) & {$[35,37,49]$} & 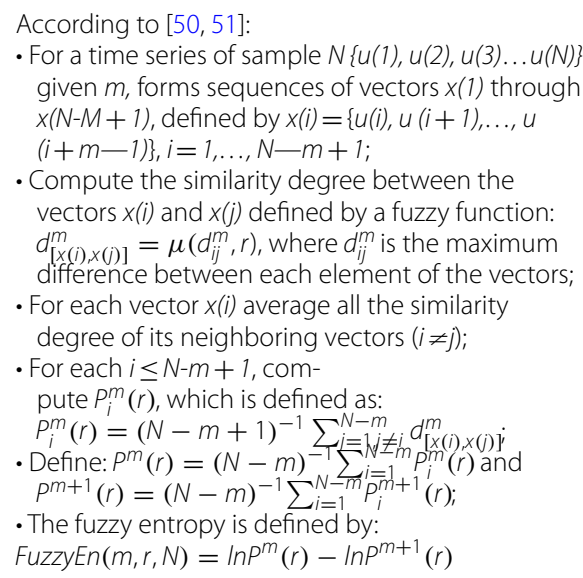 \\
\hline Variance (VAR) & {$[35-37]$} & $\begin{array}{l}\mathrm{VAR}=\sigma^{2}=\frac{1}{N-1} \sum_{n=1}^{N}(x(n)-\bar{x})^{2} \\
\bar{x} \text {-average of the samples }\end{array}$ \\
\hline Range (RANGE) & {$[35,52]$} & $\begin{array}{l}\text { Difference between the maximum and minimum } \\
\text { value of the signal }\end{array}$ \\
\hline Range interquartile (Int/A) & {$[35,53,54]$} & $\begin{array}{l}\text { IntIA }=Q_{3}-Q_{1} \\
Q_{3} \text {-third quartile; } \\
Q_{1} \text { - first quartile }\end{array}$ \\
\hline Skewness (Skewness) & {$[41,45,55]$} & Skewness $=\frac{\frac{1}{n} \sum_{n=1}^{N}(x(n)-\bar{x})^{3}}{\sigma^{3}}$ \\
\hline Kurtosis (Kurtosis) & {$[41,45,55]$} & Kurtosis $=\frac{\frac{1}{n} \sum_{n=1}^{N}(x(n)-\bar{x})^{4}}{\sigma^{4}}$ \\
\hline
\end{tabular}

\section{Features selection}

The features used to feed the classifiers were selected using the software $\mathrm{R}$ package, CORElearn, and the attrEval function using the ReliefF estimator and with $k=1$. The classifiers were fed with $10 \%$ of the most important features, then $20 \%$ of the most important features, and so on, in incremental steps of $10 \%$ until all the features are added for calculating the metrics (sensitivity, specificity, precision, and accuracy).

\section{Classifiers}

After the extraction of the features, the data were normalized using the $Z$-score technique. This technique is used in order that each feature is presented on the same scale in a dimensionless form. The equation for the $Z$-score is given by Eq. (2):

$$
\mathrm{ZS}=\left(\frac{\text { value }-\mu}{\sigma}\right),
$$

where $\mu$ is the mean of the features and $\sigma$ its standard deviation.

The classifiers used were:

- KNN;

- SVM;

- RF; 
Table 7 Confusion matrix

\begin{tabular}{lll}
\hline & Positive result & $\begin{array}{l}\text { Negative } \\
\text { result }\end{array}$ \\
\hline Actual positive & TP & FN \\
Actual negative & FP & TN \\
\hline
\end{tabular}

- NB.

These classifiers were chosen due to their use in various studies [56, 57].

Simulations were performed to identify the best parameters for the classifiers. In the KNN method, there exist proposals in the literature for the adoption of the $\mathrm{K}$ value, as being the square root of the size of the training set, but in our simulations, the value of $k=3$ offered the best results. In regard to the remaining classifiers, the best results for Random Forest were obtained using 120 trees, for the SVM, the polynomial kernel will be used, and for the classifier Naive Bayes, the kernel (normal) predictor will be used.

\section{Statistical analysis}

To evaluate the performance of a classifier, a confusion matrix (Table 7) was built considering the true positives (TP), false positives (FP), true negatives (TN), and false negatives (FN). Metrics for performance, sensitivity, specificity, precision, and accuracy (Eqs. 3-6, respectively), were computed for each classifier

$$
\begin{aligned}
& \text { Sensitivity }=\frac{\mathrm{TP}}{\mathrm{TP}+\mathrm{FN}} \\
& \text { Specificity }=\frac{\mathrm{TN}}{\mathrm{TN}+\mathrm{FP}} \\
& \text { Precision }=\frac{\mathrm{TP}}{\mathrm{TP}+\mathrm{FP}} \\
& \text { Accuracy }=\frac{\mathrm{TP}+\mathrm{TN}}{\mathrm{TP}+\mathrm{TN}+\mathrm{FP}+\mathrm{FN}} .
\end{aligned}
$$

The data from the subjects were randomly divided into training (about 50\%) and testing (about 50\%) sets. Thus, the data from the 14 subjects were allocated to training and the data from the remaining 13 subjects were reserved for testing. We used tenfold cross-validation to evaluate the classification performance. The R Project for Statistical Computing was used to conduct data analyses.

\section{Abbreviations}

PD: Parkinson disease; RF: Random forest; SVM: Support vector machine; KNN: K-nearest neighbor; NB: Naive Bayes; UPDRS: Unified Parkinson's Disease Rating Scale; IMU: Inertial measurement unit; HERC: Human Research Ethics Committee; NERC: National Ethics Research Committee; HCU-UFU: Hospital de Clínicas of Uberlândia of the Federal University of Uberlândia; SPD: Parkinson disease group; SH: Healthy group; M: Male; F: Female; R: Right; L: Left; SD: Standard deviation; TREMSEN: Precise Tremor Sensing Technology; NIATS: Center for Innovation and Technological Evaluation in Health; TP: True positives; FP: False positives; TN: True negatives; FN: False negatives; RMS: Root mean square; MAV: Mean absolute value; MAVFD: Mean absolute value of the first difference; MAVSD: Mean absolute value of the second difference; FMEAN: 
Mean frequency; ZC: Zero crossing; FPEAK: Peak frequency; F50: Median frequency; Power3.5_7.5: Power in frequency band 3.5-7.5 Hz; ApEn: Approximate entropy; FuzzyEn: Fuzzy entropy; VAR: Variance; Int|A: Range interquartile.

\section{Supplementary Information}

The online version contains supplementary material available at https://doi.org/10.1186/s12938-021-00888-2.

Additional file 1: Figure S1. A typical accelerometer signal from one of the volunteers. Where: a) Signal on the $X$-axis. b) Signal on the Y-axis. c) Signal on the Z-axis. d) Resultant signal (blue) and manual pulse (red). Figure S2. A typical gyroscope signal from one of the volunteers. Where: a) Signal on the X-axis. b) Signal on the Y-axis. c) Signal on the Z-axis. d) Resultant signal (blue) and manual pulse (red). Figure S3. A typical magnetometer signal from one of the volunteers. Where: a) Signal on the X-axis. b) Signal on the Y-axis. c) Signal on the Z-axis. d) Resultant signal (blue) and manual pulse (red).

\section{Acknowledgements}

The present work was carried out with the support of the National Council for Scientific and Technological Development (CNPq), Coordination for the Improvement of Higher Education Personnel (CAPES - Program CAPES/DFATD88887.159028/2017-00, Program CAPES/COFECUB-88881.370894/2019-01), along with the Foundation for Research Support of the State of Minas Gerais (APEMIG-APQ-00942-17). A. O. Andrade is a fellow of CNPq, Brazil (304818/2018-6). M. F. Vieira is a fellow of CNPq, Brazil (306205/2017-3). A. A. Pereira is a fellow of CNPq, Brazil (310911/2017-6).

\section{Authors' contributions}

LBP wrote and contributed to the analysis and interpretation of data. BCC and APSPBS wrote the manuscript, collected and analyzed data, performed the experiment, and contributed to the analysis and interpretation of data. VCD and MFV wrote the manuscript and discussed the results. AOA wrote the manuscript, and contributed to its analysis and to the interpretation of data. AAP wrote the manuscript, discussed the results, as well as contributed to its conception, design, analysis, and the interpretation of data. All authors read and approved the final manuscript.

\section{Funding}

This work is sponsored by the National Council for Scientific and Technological Development, the Foundation for Research Support of the State of Minas Gerais, and the Coordination for Improvement of Higher Education Personal.

\section{Availability of data and materials}

The datasets generated in the current study are not publicly available due to the ethical restrictions preventing public sharing of data. A non-identified set may be requested after approval from the Review Board of the Institution. Requests for the data may be sent to the corresponding author.

\section{Declarations}

\section{Ethics approval and consent to participate}

This study was approved by the Human Research Ethics Committee (HERC — n' 270.782) of the Universidade Federal de Uberlândia, and by the National Ethics Research Committee (NERC - no 361.526) of the National Health Council.

\section{Consent for publication}

All subjects signed an approved informed consent after the study procedures had been explained and consented to its publication.

\section{Competing interests}

The authors declare that they have no competing interests.

\section{Author details}

${ }^{1}$ Postgraduate Program in Electrical and Biomedical Engineering, Faculty of Electrical Engineering, Centre for Innovation and Technology Assessment in Health, Federal University of Uberlândia, Uberlândia, Brazil. ${ }^{2}$ Department of Information Technology, UNA Uberlândia University Center, Uberlândia, Brazil. ${ }^{3}$ Department of Morphology, Mineiros University Center, Mineiros, Brazil. ${ }^{4}$ Faculty of Physical Education and Physiotherapy, Federal University of Uberlândia, Uberlândia, Brazil. ${ }^{5}$ Bioengineering and Biomechanics Laboratory, Federal University of Goiás, Goiânia, Brazil.

Received: 4 March 2021 Accepted: 11 May 2021

Published online: 22 May 2021

\section{References}

1. Massano J, Bhatia KP. Clinical approach to Parkinson's disease: features, diagnosis, and principles of management. Cold Spring Harb Lab Press. 2012;2:1-16.

2. Tanner CM, Goldman SM. Epidemiology of Parkinson's disease. Neurol Clin. 1996;14:317-35.

3. Maserejian N, Vinikoor-Imler L, Dilley A. Estimation of the 2020 Global Population of Parkinson's Disease (PD) [abstract]. Movement Disorder. 2020; 35 Suppl 1. https://www.mdsabstracts.org/abstract/estimation-of-the-2020global-population-of-parkinsons-disease-pd/. Accessed 13 May 2021 
4. Bronstein J, Carvey P, Chen H, Cory-Slechta D, DiMonte D, Duda J, et al. Meeting report: consensus statement-Parkinson's disease and the environment: collaborative on health and the environment and Parkinson's Action Network (CHE PAN) conference 26-28 June 2007. Environ Health Perspect. 2009;117:117-21.

5. Sharma A, Szeto K, Desilets AR. Efficacy and safety of deep brain stimulation as an adjunct to pharmacotherapy for the treatment of Parkinson disease. Ann Pharmacother. 2012;46:248-54.

6. Lenz FA, Kwan HC, Martin RL, Tasker RR, Dostrovsky JO, Lenz YE. Single unit analysis of the human ventral thalamic nuclear group: tremor-related activity in functionally identified cells. Brain. 1994;117:531-43.

7. Teskey WJE, Elhabiby M, El-Sheimy N. Inertial sensing to determine movement disorder motion present before and after treatment. Sensors. 2012;12:3512-27.

8. Rizzo G, Copetti M, Arcuti S, Martino D, Fontana A, Logroscino G. Accuracy of clinical diagnosis of Parkinson disease. Neurology. 2016;86:566-76.

9. Mancini M, El-Gohary M, Pearson S, Mcnames J, Schlueter H, Nutt JG, et al. Continuous monitoring of turning in Parkinson's disease: Rehabilitation potential. NeuroRehabilitation. 2015;37:3-10.

10. Hoehn MM, Yahr MD. Parkinsonism: onset, progression, and mortality. Neurology. 1967;17(5):427-42.

11. Caslake R, Moore JN, Gordon JC, Harris CE, Counsell C. Changes in diagnosis with follow-up in an incident cohort of patients with parkinsonism. J Neurol Neurosurg Psychiatry. 2008;79:1202-7.

12. Kuhner A, Schubert T, Cenciarini M, Wiesmeier IK, Coenen VA, Burgard W, et al. Correlations between motor symptoms across different motor tasks, quantified via random forest feature classification in Parkinson's disease. Front Neurol. 2017;8:1-9.

13. Goetz CG, Fahn S, Martinez-Martin P, Poewe W, Sampaio C, Stebbins GT, et al. Movement disorder society-sponsored revision of the unified Parkinson's disease rating scale (MDS-UPDRS): process, format, and clinimetric testing plan. Mov Disord. 2007;22:41-7.

14. De Venuto D, Annese VF, Defazio G, Gallo VL, Mezzina G. Gait analysis and quantitative drug effect evaluation in Parkinson disease by jointly EEG-EMG monitoring. Proceedings of the 12th international conference on design \& technology of integrated systems In: Nanoscale Era (DTIS). 2017; pp 1-6. https://doi.org/10.1109/DTIS.2017.7930171.

15. Salarian A, Russmann H, Vingerhoets FJG, Dehollain C, Blanc Y, Burkhard PR, et al. Gait assessment in Parkinson's disease: Toward an ambulatory system for long-term monitoring. IEEE Trans Biomed Eng. 2004;51:1434-43.

16. Lee HJ, Kim SK, Park H, Kim HB, Jeon HS, Jung YJ, et al. Clinicians'tendencies to under-rate Parkinsonian tremors in the less affected hand. PLOS ONE. 2015;10:1-9.

17. Regnault A, Boroojerdi B, Meunier J, Bani M, Morel T, Cano S. Does the MDS-UPDRS provide the precision to assess progression in early Parkinson's disease? Learnings from the Parkinson's progression marker initiative cohort. J Neurol. 2019;266:1927-36. https://doi.org/10.1007/s00415-019-09348-3.

18. Niazmand K, Tonn K, Kalaras A, Kammermeier s, Boetzel K, Mehrkens J-H, Lueth TC. A measurement device for motion analysis of patients with Parkinson's disease using sensor based smart clothes. Proceedings of the 5th international ICST conference on pervasive computing technologies for healthcare. 2011; pp 9-16. https://doi.org/ 10.4108/icst.pervasivehealth.2011.246014.

19. Ai L, Wang J, Yao R. Classification of parkinsonian and essential tremor using empirical mode decomposition and support vector machine. Digit Signal Process A Rev J. 2011;21:543-50.

20. Woods AM, Nowostawski M, Franz EA, Purvis M. Parkinson's disease and essential tremor classification on mobile device. Pervasive Mob Comput. 2014;13:1-12.

21. Palmes P, Ang WT, Widjaja F, Tan LCS, Au WL. Pattern mining of multichannel sEMG for tremor classification. IEEE Trans Biomed Eng. 2010;57:2795-805.

22. Jalloul N, Porée F, Viardot G, L'Hostis P, Carrault G. Activity recognition using multiple inertial measurement units. Innov Res Biomed Eng. 2016;37:180-6.

23. Ghanad NK, Ahmadi S. Combination of PSO algorithm and naive bayesian classification for Parkinson disease diagnosis. Adv Comput Sci an Int J. 2015;4:119-25.

24. Deuschl G, Lauk M, Timmer J. Tremor classification and tremor time series analysis. Chaos. 1995;5:48-51.

25. Deuschl G, Bain P, Brin M, Agid Y, Benabid L, Benecke R, et al. Consensus statement of the Movement Disorder Society on tremor. Mov Disord. 1998;13:2-23.

26. Zhou T. Oscillation amplitude. In: Dubitzky W, Wolkenhauer O, Cho K-H, Yokota H (eds) Encyclopedia of systemsbiology. Springer, New York, 2013; p. 1616. https://doi.org/10.1007/978-1-4419-9863-7_523.

27. Afsar O, Tirnakli U, Kurths J. Entropy-based complexity measures for gait data of patients with Parkinson's disease. Chaos An Interdiscip J Nonlinear Sci. 2016;26:023115.

28. Patel S, Lorincz K, Hughes R, Huggins N, Growdon J, Standaert D, et al. Monitoring motor fluctuations in patients with Parkinson's disease using wearable sensors. IEEE Trans Inf Technol Biomed. 2009;13:864-73.

29. Machado ARP, Zaidan HC, Paixão APS, Cavalheiro GL, Oliveira FHM, Júnior JAFB, et al. Feature visualization and classification for the discrimination between individuals with Parkinson's disease under levodopa and DBS treatments. Biomed Eng Online. 2016;15:169.

30. Rabelo AG, Neves LP, Paixão APS, Oliveira FHM, de Souza LAPS, Vieira MF, et al. Objective assessment of bradykinesia estimated from the wrist extension in older adults and patients with Parkinson's disease. Ann Biomed Eng. 2017:45:2614-25.

31. Juutinen M, Wang C, Zhu J, Haladjian J, Ruokolainen J, Puustinen J, et al. Parkinson's disease detection from 20-step walking tests using inertial sensors of a smartphone: machine learning approach based on an observational casecontrol study. PLoS ONE. 2020;15:1-19. https://doi.org/10.1371/journal.pone.0236258.

32. Khoury N, Attal F, Amirat Y, Oukhellou L, Mohammed S. Data-driven based approach to aid Parkinson's disease diagnosis. Sensors (Switzerland). 2019;19:1-27.

33. de Araújo ACA, da Santos EGR, de Sá KSG, Furtado VKT, Santos FA, de Lima RC, et al. Hand resting tremor assessment of healthy and patients with Parkinson's disease: an exploratory machine learning study. Front Bioeng Biotechnol. 2020;8:1-13.

34. Jeon H, Lee W, Park H, Lee HJ, Kim SK, Kim HB, et al. Automatic classification of tremor severity in Parkinson's disease using a wearable device. Sensors (Switzerland). 2017;17:1-14. 
35. Gruss S, Treister R, Werner P, Traue HC, Crawcour S, Andrade A, et al. Pain intensity recognition rates via biopotential feature patterns with support vector machines. PLoS ONE. 2015;10:1-14.

36. Phinyomark A, Hirunviriya S, Limsakul C, Phukpattaranont P. Evaluation of EMG feature extraction for hand movement recognition based on euclidean distance and standard deviation. In: ECTI-CON 2010-2010 ECTI international conference on electrical engineering computer telecommunication information technology. 2010; pp. 856-60.

37. Zhang Y, Yu J, Xia C, Yang K, Cao H, Wu Q. Research on GA-SVM based head-motion classification via mechanomyography feature analysis. Sensors (Switzerland). 2019;19:1-12.

38. Garcia MAC, Vieira TMM. Surface electromyography: why, when and how to use it. Rev Andaluza Med del Deport. 2011:4:17-28

39. Englehart K, Hudgins B. A robust, real-time control scheme for multifunction myoelectric control. IEEE Trans Biomed Eng. 2003;50:848-54.

40. Vyzas E, Picard RW. Affective pattern classification. In: Proceedings of the AAAl1998 fall symposium emotional intelligent: the tangled knot cognition. 1998; pp. 176-182.

41. Khalili Z, Moradi MH. Emotion recognition system using brain and peripheral signals: Using correlation dimension toimprove the results of EEG. In: Proceedings of the international joint conference on neural networks. 2009;pp. 1571-5. https://doi.org/10.1109/IJCNN.2009.5178854.

42. Khanam F, Ahmad M. Frequency based EMG power spectrum analysis of Salat associated muscle contraction. In: ICEEE2015-1st international conference on electrical and electronic engineering. 2016; pp. 161-4. https://doi.org/ 10.1109/CEEE.2015.7428245.

43. Gross V, Dittmar A, Penzel T, Schüttler F, Von Wichert P. The relationship between normal lung sounds, age, and gender. Am J Respir Crit Care Med. 2000;162:905-9.

44. McDonald AC, Mulla DM, Keir PJ. Using EMG amplitude and frequency to calculate a multimuscle fatigue score and evaluate global shoulder fatigue. Hum Factors. 2019;61:526-36.

45. Ribeiro L, De PM, Pereira AA, Fernanda M, De AS, Cavalheiro GL, et al. Analysis of the relationship between EEG signal and aging through Linear Discriminant Analysis (LDA). Res Biomed Eng. 2012;28:155-68.

46. Butt AH, Rovini E, Esposito D, Rossi G, Maremmani C, Cavallo F. Biomechanical parameter assessment for classification of Parkinson's disease on clinical scale. Int J Distrib Sens Networks. 2017;13:1-15.

47. Ahmad SA, Chappell PH. Moving approximate entropy applied to surface electromyographic signals. Biomed Signal Process Control. 2008;3:88-93.

48. Pincus SM. Approximate entropy as a measure of system complexity. Proc Natl Acad Sci. 1991;88:2297-301.

49. Chen W, Wang Z, Xie H, Yu W. Characterization of surface EMG signal based on fuzzy entropy. IEEE Trans Neural Syst Rehabil Eng. 2007;15:266-72.

50. Chen W, Zhuang J, Yu W, Wang Z. Measuring complexity using FuzzyEn, ApEn, and SampEn. Med Eng Phys. 2009;31:61-8.

51. Ishikawa A, Mieno H. The fuzzy entropy concept and its application. Fuzzy Sets Syst. 1979;2:113-23.

52. Cavalheiro GL, Almeida MFS, Pereira AA, Andrade AO. Study of age-related changes in postural control during quiet standing through linear discriminant analysis. Biomed Eng Online. 2009:8:35.

53. Antonelli A, Guilizzoni D, Angelucci A, Melloni G, Mazza F, Stanzi A, et al. Comparison between the airgo device and a metabolic cart during rest and exercise. Sensors. 2020;20:1-18.

54. Perez H, Tah JHM. Improving the accuracy of convolutional neural networks by identifying and removing outlier images in datasets using t-SNE. Mathematics. 2020;8:662.

55. Rizon MMM. Feature extraction methods for human emotion recognition using EEG—a study. In: Conference on Malaysia-Japan international symposium on advanced technology. 2007.

56. Haghnegahdar AA, Kolahi S, Khojastepour L, Tajeripour F. Diagnosis of tempromandibular disorders using local binary patterns. J Biomed Phys Eng. 2018;8:87-96.

57. Calil BC, Da Cunha DV, Vieira MF, De Oliveira AA, Furtado DA, Bellomo Junior DP, et al. Identification of arthropathy and myopathy of the temporomandibular syndrome by biomechanical facial features. Biomed Eng Online BioMed Central. 2020;19:1-18.

\section{Publisher's Note}

Springer Nature remains neutral with regard to jurisdictional claims in published maps and institutional affiliations.

Ready to submit your research? Choose BMC and benefit from:

- fast, convenient online submission

- thorough peer review by experienced researchers in your field

- rapid publication on acceptance

- support for research data, including large and complex data types

- gold Open Access which fosters wider collaboration and increased citations

- maximum visibility for your research: over $100 \mathrm{M}$ website views per year

At BMC, research is always in progress.

Learn more biomedcentral.com/submissions 